\title{
Preoperative Assessment and Patient Safety in Interventional Neuroradiology
}

\author{
Ayten Saracoglu* \\ Department of Anesthesiology, Science University School of Medicine, Istanbul, Turkey
}

*Corresponding author: Ayten Saracoglu, Department of Anesthesiology Science, University School of Medicine, Florence Nightingale Hospital Abide-I Hurriyet Cad. No. 166, Sisli Caglayan Istanbul, Turkey, Tel: +902123756565; E-mail: anesthesiayten@gmail.com

Rec date: Jul 7, 2014; Acc date: Jul 8, 2014; Pub date: Jul 10, 2014

Copyright: @ 2014 Saracoglu A. This is an open-access article distributed under the terms of the Creative Commons Attribution License, which permits unrestricted use, distribution, and reproduction in any medium, provided the original author and source are credited.

\section{Editorial}

Performing interventional neuroradiology procedures outside the operating room (non-OR) is not only difficult but also risky [1]. Therefore, the procedure should be carefully planned. Many interventional neuroradiology procedures take place in remote locations away from the operating room. These locations may not be even on the same floor. Other disadvantages of non-OR interventional neuroradiology are the need to work in environments with reduced light level and exposure to intense ionizing radiation.

Besides normal preanaesthetic evaluation, a careful neurological examination focused on Glascow Coma Scale (GCS) is required in neuroradiology procedures to identify existing and known deficits [2]. Pupil size and reactivity should be specified and recorded. Patients' need for intensive care, arterial blood pressure and other baseline parameters regarding cardiovascular and renal reserve and coagulation should be determined. Additionally, the patients' history of previous angiography and allergy to contrast agents and protamine should be recorded. Sedative premedication should be avoided.

Airway manipulation may be difficult during the procedure due to the head position decided for monitoring. Airway safety should be carefully ensured. History of previous radiological procedures and reaction to contrast agents is also significant. As in all non-OR procedures performed under anesthesia the communication between the anesthesiologist and radiologist is significant for optimal patient care. Some procedures require postoperative neurological assessment in awake patients. Anesthetic recovery should be fast to perform neurological assessment in the shortest time possible. There is not sufficient evidence suggesting the superiority of intravenous or inhaled anesthesia over each other [3]. Under clinical conditions, all volatile agents have the potential to increase cerebral blood flow, cerebral blood volume, and intracranial pressure [4]. As nitrous oxide elevates cerebral blood flow and intracranial pressure, and increases the consequences of air embolism, it should be avoided. Cerebral blood flow, intracranial pressure and cerebral metabolic rate of oxygen can be reduced with a Total Intravenous Anesthesia (TIVA) technique incorporating propofol and a short-acting opioid. However, it has been reported that sevoflurane is associated with earlier recovery than propofol, for long duration interventional neuroradiology procedures [5]. As it has low potential for increasing cerebral blood flow and intracranial pressure, sevoflurane can be preferred as inhalation agent. Sevoflurane also provides easier postoperative neurological assessment by ensuring faster recovery compared to isoflurane.

Whatever anesthetic technique is selected, standard anesthesia monitors are utilized. Monitoring should include pulse oximetry, Electrocardiogram (ECG) and capnography. Presence of medical comorbidities may require measurement of central pressures. It is recommended to install two intravenous catheters. One is used for infusion of medication while the other is used for liquids and bolus doses. Extension line can be applied in case that vascular access cannot be achieved. If intraoperative and postoperative control of blood pressure is considered significant for attempts requiring manipulation of blood pressure, radial artery catheter should be utilized for monitoring. However, if there is no need for postoperative blood pressure control, anesthesiologist may use the arterial line provided by the radiologist.

Many procedures require urinary catheterization. It is essential not only for liquid management but also for patient comfort. Significant amount of heparinized flush solutions and contrast agents are used during catheterization. Diuretics, such as mannitol and furosemide, may be also required during the procedure. Hypothermia may occur within the process. Therefore, heating blankets should be used to maintain heating and temperature measurement should be routinely performed.

In interventional procedures, patients receive significant amounts of contrast agent. The amount may reach $150-250 \mathrm{~mL}$ in some patients. Plasma creatinine levels and glomerular filtration rate should be evaluated in the preoperative period.

Thrombosis is a complication of endovascular procedures. Thus, heparin is commonly used in these cases. The aim is to keep the activated clotting time (ACT) 2-2.5-fold over basal levels. In general, a baseline ACT is obtained. Afterwards, $70 \mathrm{IU} / \mathrm{kg}$ heparin is given intravenously to increase ACT approximately 2 to 3 times above the baseline value. ACT should be assessed with hourly monitoring, and additional dose of heparin should be given if needed. Although rare, one should be prepared for major blood loss due to injuries in major vessels.

Besides its use in some balloon test occlusions to reduce blood flow in feeding arteries, controlled hypotension is often applied during AVM embolization. Agents like esmolol, labetalol, and sodium nitroprusside are useful in such situations. Controlled hypotension is performed during cerebral ischemia in order to maximize collateral flow. Phenylephrine is generally used, both as a bolus and as an infusion titrated to increase systolic blood pressure 30\%-40\% above baseline values. In these patients, close monitoring of ECG parameters for signs of myocardial ischemia is critical. Also, necessary precautions should be taken to avoid coughing and bucking. Intracranial pressure should be checked, and cerebral perfusion pressure should be appropriately maintained during anesthesia.

Patients are exposed to high doses of ionizing radiation during the procedure. Therefore, pregnancy must be excluded in female patients aged between 15 and 55 years. Patients with cerebral aneurysm may present with subarachnoid hemorrhage or cranial nerve palsies. Endovascular coiling can be safely performed in patients suffering from aneurysm rupture. In case of any hemodynamic instability 
during the procedure, radiologist should be warned to take care of the aneurysm rupture. Blood pressure should be decreased if aneurysm rupture develops.

While $5 \%$ to $10 \%$ of arteriovenous malformations develop following subarachnoid hemorrhage, most present with seizures, headache, and focal neurologic findings. Anesthesia should be terminated smoothly avoiding from coughing and hypertension. Otherwise, cerebral edema may develop. Minimal postoperative pain is observed as in several radiological interventions. However, severe postoperative headache may be indicative of intracranial hemorrhage.

When interventional techniques are utilized for management of carotid occlusive disease, bradycardia or asystole may develop due to carotid body stimulation during angioplasty. It is challenging to prevent them before development; however, glycopyrrolate or atropine may be useful. Postoperative hypertension should be avoided to prevent cerebral reperfusion injury.

Patients suffering from aneurysmal subarachnoid hemorrhage should be monitored for increased intracranial pressure, cerebral ischemia and hydrocephalus. General anesthesia is preferred for coiling of cerebral aneurysm, because the lack of movement and during procedure reduces the incidence of perforation. Thrombus may develop on the catheter, guide wire, or coil during or after placement of the coil. The overall incidence of thromboembolic complication is 2.5$5 \%$ [6]. Hyperactivity of the sympathetic system following subarachnoid hemorrhage, and massive catecholamine release may lead to critical ECG changes, arrhythmias, and left ventricular failure. Unconscious patients are prone to aspiration and pneumonia. Neurogenic pulmonary edema may form following Subarachnoid Hemorrhage (SAH) both in cardiac and non-cardiac mechanisms. Due to syndrome of inappropriate antidiuretic hormone or cerebral saltwasting syndrome, hyponatremia is seen in $10-34 \%$ of individuals with $\mathrm{SAH}$. As blood pressure $>160 \mathrm{mmHg}$ may lead to the rupture of an aneurysm, it should be carefully monitored. Vasodilators, such as hydralazine, glyceryl trinitrate and sodium nitroprusside, increase intracranial pressure and cerebral blood volume and lead to ischemia by impairing cerebral perfusion. Calcium channel blockers reduce the incidence of vasospasm. Vasospasm requires the triple- $\mathrm{H}$ therapy: hypertension, hypervolemia and hemodilution. Blood pressure should be decreased to reduce blood flow in the feeding artery before the injection of glue in AVM [7].

Preoperative embolization is applied for vascular tumors including meningiomas, glomus tumor, and juvenile nasopharyngeal angiofibroma. The purpose of embolization is to minimize blood loss and facilitate dissection by reducing tumor vascularity.

Moderate hypotension is recommended to prevent cerebral edema and hemorrhage after AVM embolization. The mean arterial pressure should be kept $15-20 \%$ below the baseline value during the first 24 hours. Due to their minimal effect on cerebral physiology, antihypertensive agents, such as labetalol or esmolol, can be used to control blood pressure. On the other hand, the mean arterial pressure should be kept above $20-30 \%$ to maintain cerebral perfusion pressure in patients with occlusive conditions or vasospasm. This can be achieved with phenylephrine or norepinephrine. Nimodipine is used up to 3 weeks in patients with aneurysmal subarachnoid hemorrhage (aSAH). Most patients carry on aspirin treatment $(75 \mathrm{mg})$ during 3 months. Due to the use of contrast agents and anesthetics, nausea and vomiting may be observed in patients after the procedure.

Patients requiring interventional neuroradiology may come directly from the intensive care unit, and safe transfer is crucial for them. Safe transfer includes maintenance of the external ventricular drainage catheter or intracranial pressure monitoring device.

\section{References}

1. Metzner J, Domino KB (2010) Risks of anesthesia or sedation outside the operating room: the role of the anesthesia care provider. Curr Opin Anaesthesiol 23: 523-531.

2. Czapiga B, Koźba-Gosztyła M, Jarmundowicz W, Szczepański T. (2013) Surgical management in patients with aneurysmal subarachnoid hemorrhage. The outcomes in the paradigm shift period. Adv Clin Exp Med 22: 539-547.

3. Patel S, Appleby I (2013) Anaesthesia for interventional neuroradiology. Anaesthesia \& intensive care medicine 14: 387-390.

4. Bösel J, Purrucker JC, Nowak F, Renzland J, Schiller P, et al. (2012) Volatile isoflurane sedation in cerebrovascular intensive care patients using AnaConDa $\left({ }^{\oplus}\right)$ : effects on cerebral oxygenation, circulation, and pressure. Intensive Care Med 38: 1955-1964.

5. Castagnini HE, van Eijs F, Salevsky FC, Nathanson MH (2004) Sevoflurane for interventional neuroradiology procedures is associated with more rapid early recovery than propofol. Can J Anaesth 51: 486-491.

6. Schulenburg E, Matta B (2011) Anaesthesia for interventional neuroradiology. Curr Opin Anaesthesiol 24: 426-432.

7. Orlowski P, Summers P, Noble JA, Byrne J, Ventikos Y (2012) Computational modelling for the embolization of brain arteriovenous malformations. Med Eng Phys 34: 873-881. 